\title{
Controlling the optics of quantum dots with nanomechanical strain
}

\author{
Garnett W. Bryant,,${ }^{1, *}$ M. Zieliński, ${ }^{2}$ Natalia Malkova,,${ }^{1}$ James Sims, ${ }^{3}$ W. Jaskólski, ${ }^{2}$ and Javier Aizpurua ${ }^{4}$ \\ ${ }^{1}$ Atomic Physics Division and Joint Quantum Institute, National Institute of Standards and Technology, 100 Bureau Drive, \\ Gaithersburg, Maryland 20899-8423 \\ ${ }^{2}$ Instytut Fizyki UMK, Grudziądzka 5, 87-100 Toruń, Poland \\ ${ }^{3}$ Information Technology Laboratory, National Institute of Standards and Technology, 100 Bureau Drive, \\ Gaithersburg, Maryland 20899-8911 \\ ${ }^{4}$ Donostia International Physics Center and Centro Mixto de Física de Materiales CSIC-UPV/EHU, Paseo Manuel Lardizabal 4 , \\ Donostia-San Sebastián E-20018, Spain
}

(Received 26 August 2011; revised manuscript received 17 November 2011; published 1 December 2011)

\begin{abstract}
We show how nanomechanical strain can be used to dynamically control the optical response of self-assembled quantum dots embedded in nanomechanical bridges, giving a tool to shift electron and hole levels, manipulate mechanoexciton shape, orientation, fine-structure splitting, and optical transitions, transfer carriers between dots, and interact qubits for quantum processing. Conversely, we show how modulation of the quantum dot optical response can be used to monitor locally an applied nanomechanical strain. Atomistic tight-binding theory is used to describe the response of electrons and holes in a self-assembled quantum dot to applied nanomechanical strain. The internal strain due to the lattice mismatch, the nanomechanical strain, and the internal atomic readjustment to minimize the applied strain must all be accounted for to model correctly the strain effects. Electrons and hole levels and charge distributions can shift together or in opposite directions depending on how the strain is applied. This gives control for tailoring band gaps and optical response. The strain can also be used to transfer electrons and holes between vertically or laterally coupled dots, giving a mechanism for manipulating transition strengths and interacting qubits for quantum information processing. Applied strain can be used to manipulate the fine-structure splitting of mechanoexcitons by distorting electron and hole charge distributions and rotating hole orientation. Most importantly, nanomechanical strain reengineers both the magnitude and phase of the exciton exchange coupling to tune exchange splittings, change the phase of spin mixing, and rotate the polarization of mechanoexcitons, providing phase and energy control of excitons.
\end{abstract}

DOI: 10.1103/PhysRevB.84.235412

PACS number(s): 78.67.Hc, 73.21.La, 85.35.Be

\section{INTRODUCTION}

Semiconductor quantum dots (QDs) have attracted great interest due to their potential applications in electronics, photonics, quantum information, and high-precision measurements. Passive control of the properties of quantum dots is achieved by tailoring dot size, shape, and composition via growth. However, dynamical control of the exciton states in QDs is highly desirable for applications in nanophotonics. For example, two-photon cascade from the QD biexciton state ${ }^{1,2}$ is being studied now by many groups as a route toward entangled photon generation in scalable devices. ${ }^{3}$ However, the anisotropic exchange splitting (AES) of QD exciton states, which is induced by the asymmetry of typical as-grown self-assembled dots or the lack of inversion symmetry, inhibits entanglement of the photon pair. Annealing to reduce the as-grown structural asymmetry, ${ }^{4}$ magnetic and electric fields applied to manipulate the exciton states, ${ }^{5-10}$ and dressing the states with optical control fields ${ }^{11,12}$ are being explored as ways to modify and reduce the AES. Externally imposed nanomechanical strain ${ }^{13-20}$ provides a route to dynamically reengineer the structural symmetry of the dots. This structural reshaping would modify the electronic excitations and thereby induce or split level degeneracies, polarize optical transitions, control exchange splitting, induce entanglement, or modify coupling in closely spaced dots. These are capabilities needed to use QDs in nanophotonics and quantum-information processing. In other optically active structures, such as optomechanical cavities ${ }^{21-23}$ and rolled-up semiconductor nanotubes, ${ }^{24-28}$ induced strain would also manipulate the exciton states and modulate the optical response of the QDs.

Just as nanomechanical strain could be used to control QDs, quantum dots could, conversely, be used to probe and control nanomechanical systems. Nanomechanical systems are now being intensely studied for mass sensing, ${ }^{29-31}$ lowpower mechanical computing and memory, ${ }^{32-34}$ and energy harvesting. ${ }^{35}$ Attempts to cool vibrations of such structures to the fundamental quantum limit are being pursued aggressively to realize the quantum limit in macroscopic structures, to achieve sensing at the ultimate limit needed for quantum metrology, and to provide coherent transducers for coupling to other quantum systems. ${ }^{36-40}$ Surface acoustic waves are being used to trap carriers in dynamically created quantum dots, transport these trapped carriers, and store light. ${ }^{41-49}$ To exploit these nanomechanical deformations, one must have ways to probe and control them. It has been suggested that sideband cooling via optical absorption by QDs buried in nanomechanical structures could bring the structures to the quantum limit. ${ }^{39}$ Recent work has shown that QD levels are sensitive to the local strain produced by surface acoustic waves or mechanical deformations. ${ }^{15-18,50}$ Thus QD response could also provide a local strain gauge for nanomechanical deformations.

To exploit hybrid nanomechanical-quantum dot devices, one must develop an understanding for the physical operation of such hybrid structures. This entails understanding the coupling between internal strain due to lattice mismatch, 
externally imposed nanomechanical strain, the electron and hole states of the QDs embedded in the device, and the strained excitons (mechanoexcitons) in optically excited QDs.

To identify the effects of nanomechanical strain on the optics of QDs, we consider pyramidal InAs self-assembled QDs embedded in a GaAs nanomechanical bridge using atomistic tight-binding theory. ${ }^{20}$ The bridge is bent to simulate an external strain applied to distort the structure of the QDs. We will show that a bend in the nanomechanical structure is analogous to an electric field, inducing Stark-like electron and hole level shifts, with one to ten meV energy shifts possible. Electrons and holes can be moved vertically, along the QD growth axis, or horizontally, in the plane of the QD, depending on how the strain is applied. This behavior is correlated with the bend-induced changes in the local band profile. As a consequence, applied strain can also rearrange the levels of electrons and holes in vertically or laterally coupled dots, thereby inducing tunneling between coupled dots. Most importantly, strain-induced spatial redistribution and reorientation of the states, primarily the holes, can lead to large changes in exciton exchange splitting and to rotation of the polarization of the bright-exciton transitions. Such control is possible because the electron and hole redistribution changes the magnitude and the phase of the asymmetric exchange coupling. The phase of the spin mixing in the exciton, which defines the exciton phase, is determined directly from the phase of the asymmetric exchange coupling.

The tight-binding theory and strain model we use to study mechanically strained dots is briefly reviewed in Sec. II. In Sec. III we present our results. We begin by discussing the strain distribution in mechanically strained QDs. Next we show how electron and hole levels shift and the states distort under applied strain. We also explain how these level shifts and distortions depend on the bend-induced changes in the local band profile. Next, we present results for vertically coupled QDs and laterally coupled QDs and show that strain-induced distortion of electron and hole states can lead to interdot tunneling. Finally we discuss the effect of mechanical strain on the QD exciton to show how QD photonics can be controlled by strain. An initial discussion of some of these results was presented in Ref. 20.

\section{THEORY}

Using atomistic models is critical for an accurate description of nanomechanical-QD hybrid systems with strong confinement, atomic-scale variations in composition and shape, and significant lattice mismatch at interfaces. It is also important when mechanical strain comparable to the internal strain is applied to modify dot properties. ${ }^{51} \mathrm{We}$ use atomistic tight-binding theory ${ }^{51-53}$ for the electron and hole states that incorporates an $s p^{3} s^{*}$ orbital model, nearestneighbor coupling, spin-orbit effects, local strain due to lattice mismatch, and strain imposed by bending the structure. Relaxation of local strain and imposed strain are both important, so we include them on an equal footing via atomistic valence force field theory. Exciton states are determined using a configuration-interaction treatment of the electron-hole coupling. ${ }^{54-56}$ An atomistic model is essential for describing accurately the effects of AES. ${ }^{57-59}$ AES can arise when the

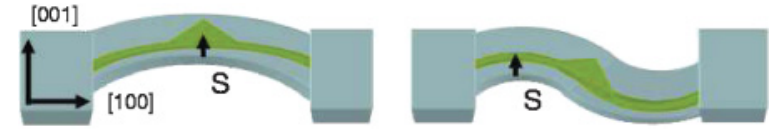

FIG. 1. (Color online) Schematic showing the bend applied to an InAs QD embedded in a GaAs nanobridge for (left) a symmetric (biaxial deformation) bend with the QD at an antinode, and (right) an antisymmetric, vertical shearing bend with the QD at a node. $S$ is the bend amplitude for clamped ends.

QD has geometrical asymmetry. ${ }^{60}$ However, AES arises even when the QD has in-plane geometrical symmetry, because the atomistic structure provides symmetry breaking. ${ }^{57,58}$

To model the nanomechanical strain, we consider an InAs QD embedded in a GaAs nanomechanical bridge, as shown in Fig. 1. The bridge is clamped at each end and bent along [100] by shifting the bridge vertically along [001], as indicated. After the structure is bent, the surface of the bridge is held fixed while all atoms inside the bridge are allowed to relax to minimize the imposed strain and the lattice-mismatch strain. Other boundary conditions could be applied, and each should be investigated to understand completely how the strain would be distributed across the bridge. We consider maximum bend amplitudes $S$ which produce a beam elongation of 0.25 percent or less and internal lattice shifts that are a tenth of the relaxation due to lattice mismatch. For the simulations presented here, the bridge is $80 \mathrm{~nm}$ wide in the lateral directions and $25 \mathrm{~nm}$ thick with 10 million atoms. A small, square-based pyramidal QD (height $3 \mathrm{~nm}$, base $7 \mathrm{~nm}$ ) with the wetting layer are located near the middle of the bridge. Because we consider a structurally symmetric QD, the AES is induced by atomistic symmetry breaking. We consider two bends: one that is symmetric about the dot that distorts the biaxial deformation induced by the lattice mismatch and the other that is antisymmetric about the dot providing a vertical shear, as shown in Fig. 1. Dots at other positions along the bridge would experience a combination of these effects. Dots closer to the top or bottom of the bridge would feel a different strain distribution. The bend is chosen along [100] to focus on the effects of the mechanical deformation rather than any piezoelectric effects that can arise for other bend directions. For a small QD, piezoelectric effects are also small. ${ }^{52}$ We ignore these piezoelectric effects here.

\section{RESULTS}

\section{A. Strain}

To begin, we discuss how strain is distributed in a mechanically strained QD. Strain due to lattice mismatch, which is inherent in InAs/GaAs QDs, the applied mechanical strain, and the resulting relaxation of the strains are all important and must be included to model the QD response to mechanical strain. Figure 2 compares strain in bent bridges with strain in an unbent, flat bridge. In a flat bridge, there is large lateral compression of the InAs lattice in the QD due to the 7 percent lattice mismatch. In the vertical direction, the lattice constant in the dot approaches the InAs lattice constant (the lattice constants are $a_{\mathrm{GaAs}} \equiv a=0.565 \mathrm{~nm}$ and $a_{\mathrm{InAs}}=0.606 \mathrm{~nm}$ ), especially in the wetting layer and near the bottom of the dot. Near the top of the dot, the compression due to lattice mismatch is larger. 

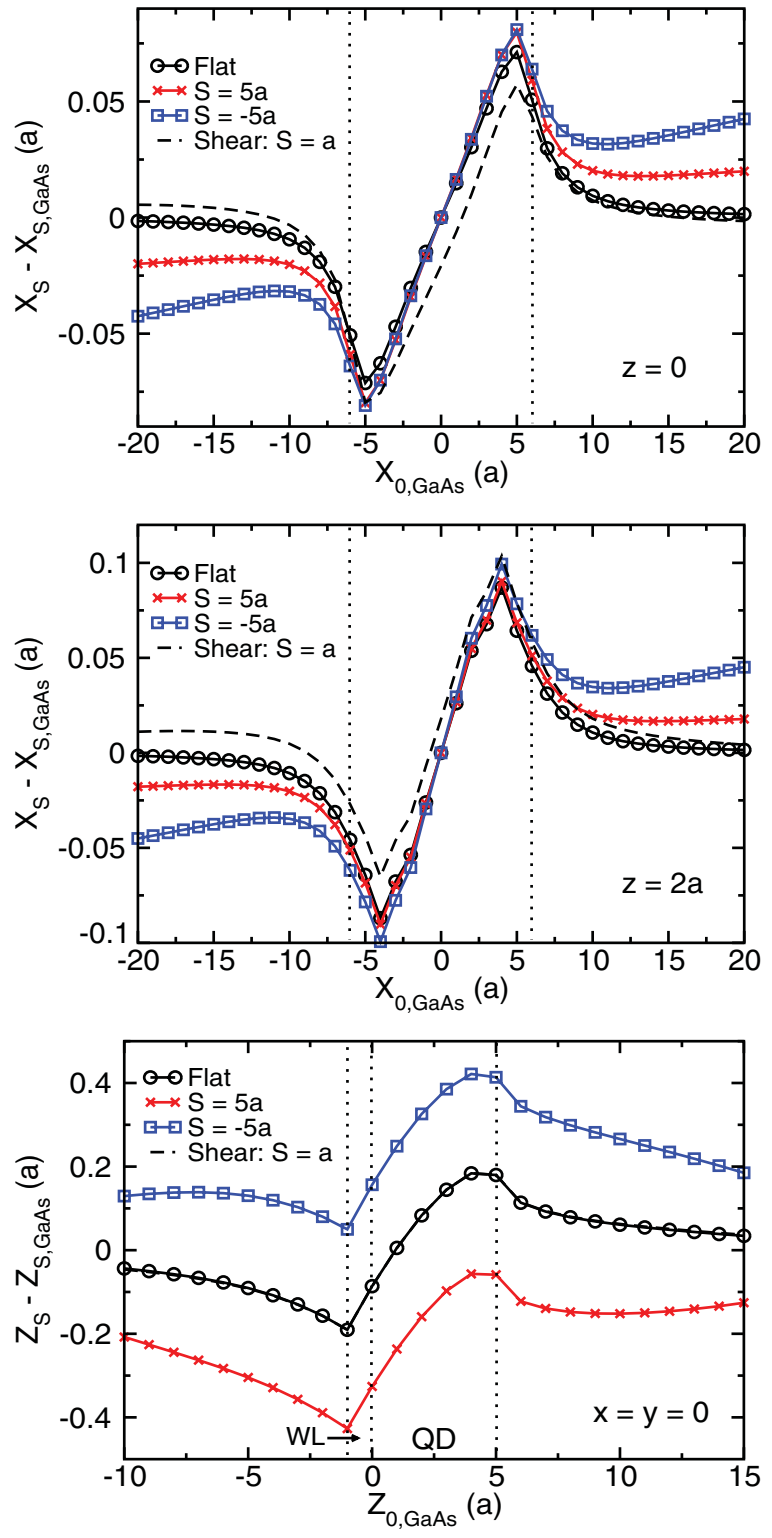

FIG. 2. (Color online) The lattice position in a bent, relaxed bridge $\left(x_{S}\right.$ and $\left.z_{S}\right)$ for a flat bridge $(S=0)$, for bridges with biaxial deformation ( $S=5 a$ and $-5 a$, with $a$ the GaAs lattice constant) and for a sheared bridge $(S=a)$, shown relative to the lattice positions in a bent, unrelaxed bridge $\left(x_{S, \text { GaAs }}\right.$ and $\left.z_{S, \text { GaAs }}\right)$ with all atoms at GaAs lattice positions. The change of $x$ at the top of the wetting layer $(z=0)$ and inside the $\operatorname{dot}(z=2 a)$ along the line $y=0$ through the center of the dot are shown. The change of $z$ along the axis of the dot $(x=y=0)$ is also shown. The outer edges of the dot are indicated by dotted lines.

For a bend symmetric about the dot that biaxially deforms the dot $(S=5 a$ and $-5 a)$, there is a small, extra lateral expansion of the lattice near the wetting layer. Further inside the dot (i.e., above the wetting layer) the expansion is slightly greater for $S=-5 a$ than for the opposite bend. For the bend amplitudes shown, the extra lattice relaxation after bending is an order of magnitude or more smaller than the relaxation due to lattice mismatch. For a shearing bend $[S=a$ corresponds to an upward bend of the right side $(x>0)$ of the dot], there is less lateral expansion than in the unbent bridge. Near the wetting layer this is accomplished by a lateral shift of the atoms to the left. Further up into the dot, the shear shifts the atoms to the right.

In the vertical direction $(z)$, the expansion along the dot axis for a shearing bend and for the flat, unbent bridge are the same, as expected (in the bottom panel of Fig. 2 the curve with circles and the dash-dot curve are indistinguishable). However, there are differences between the vertical expansion of a flat bridge and the symmetrically bent bridge. There is an overall vertical shift of the lattice. When the bridge is pushed down, the lattice near the dot relaxes upward to undo the bend. The opposite occurs when the bridge is bent upward. This clearly indicates that the lattice in a bent bridge is not simply the bent lattice of the flat bridge. The additional internal relaxation in response to the bend is an essential component of the total relaxation. When the curves in Fig. 2 for the vertical expansion for bends symmetric about the dot are superimposed on the curve for the vertical expansion of the unbent bridge, the curves are nearly identical. However, small differences remain. These small differences help explain the energy-level shifts when the bridge is bent.

Several conclusions can be drawn. The relaxation due to lattice mismatch, the mechanically applied strain, and relaxation in response to the applied strain are all important. Internal reaction to the applied strain tries to undo a symmetrically applied strain. An applied shearing strain breaks the lateral symmetry of the dot.

\section{B. Electron and hole energies}

Figure 3 shows the shift in electron and hole levels when the bridge is bent to biaxially deform or shear the dot. Throughout, electron energies are referenced to the top of the GaAs bulk valence band. Valence level energies, also referenced to the top of the GaAs valence band, are shown in Fig. 3. Hole energies are the negative of these valence-state energies. A symmetric bend can raise or lower the electron energy levels, depending on how the bend is applied. One to ten meV shifts are possible for the bend amplitudes shown. The levels shift rigidly, suggesting that the primary effect of the bend is a spatially uniform shift of the conduction band edge in the dot. As we will show later, the change in band profile is more complicated than a uniform shift. The strain-induced change $\Delta E_{e}$ of the lowest electron level is shown in Fig. 3(e). A maximum change is achieved for $S \approx 2 a$. As will be discussed later, this maximum correlates to how the applied strain moves the electron around inside the dot, pinning the electron close to the top of the dot for $S>0$, and how the strain distorts the band profile. The hole levels shift the same way as the electron levels, increasing or decreasing by a similar amount as for electrons. However, no maximum is reached, over the range shown here, for holes. This correlates to differences in electron and hole masses, in how electrons and holes are localized in the dot, and the consequence that the hole never becomes pinned for these applied strains. For $S>0$, the change in hole energy is much larger than the change in electron energy. This also indicates that the electron is more strongly pinned by the dot confinement and less sensitive to the applied strain for $S>0$ when the strain increases the localization in the dot. 

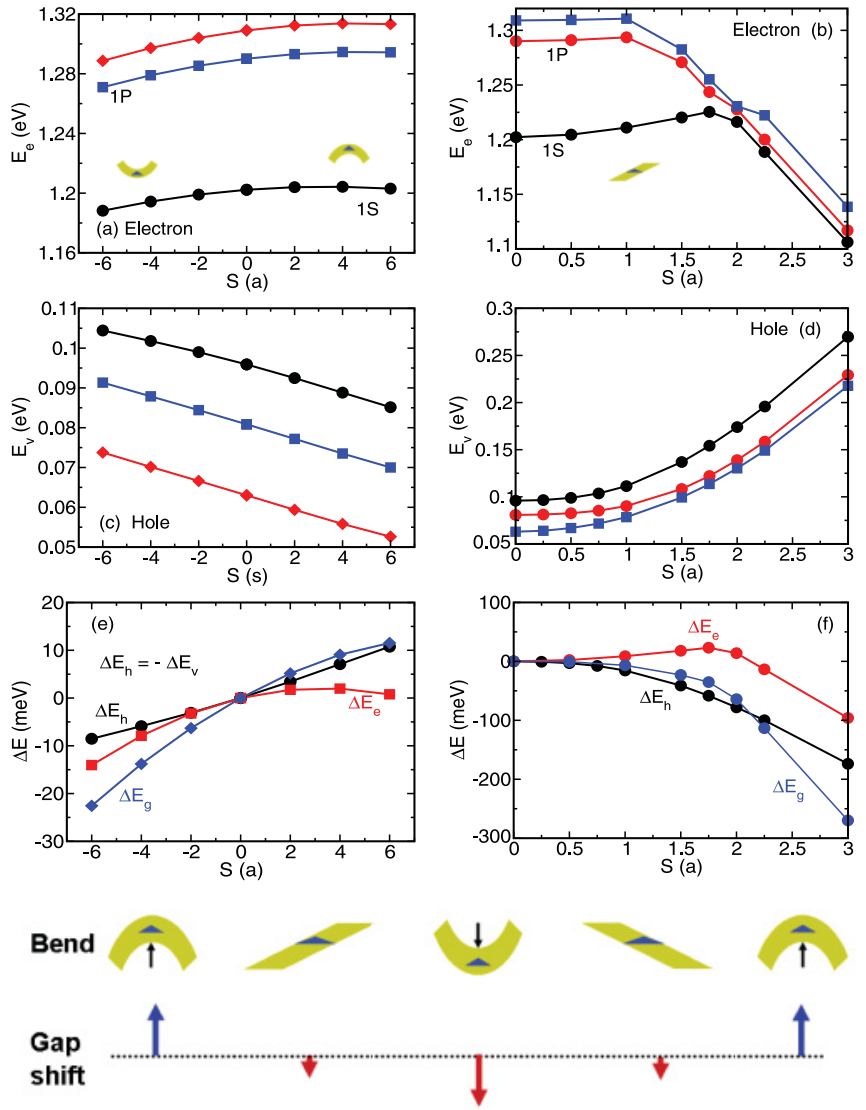

FIG. 3. (Color online) Dependence of the electron $1 S$ and $1 P$ energies $E_{e}$ on bend amplitude $S$ for (a) a biaxial deformation and (b) a shearing bend, as indicated by the schematic insets. Energy shifts for the valence (hole) levels $E_{v}\left(E_{h}=-E_{v}\right)$ at the top of the valence band are shown for the same bends [(c), (d)]. The straininduced change in the lowest electron level $\left(\Delta E_{e}\right)$, the lowest hole level $\left(\Delta E_{h}=-\Delta E_{v}\right)$, and the single-particle band gap $\left(\Delta E_{g}\right)$ due to the bends are shown in (e) and (f). The corresponding blueshift or redshift of the band gap is indicated for different bends where the lowest electron and hole states remained confined, dot states.

For an antisymmetric, shearing bend, the lowest confined electron levels increase until there is a crossing with energy levels of wetting-layer states near $S=2 a$, suggesting that the electron levels are pushed laterally out of the dot by a shearing bend. For small $S$, the shifts are in the one to ten meV range, just as for symmetric bends. For large $S$ where the level crossing occurs, much larger shifts are possible. As will be discussed, the increase in electron energy correlates to increased confinement resulting from strain-induced lateral shifts of the electron toward the dot sides. For a shearing bend, the hole energies are initially lowered by the bend, opposite to the electron level shifts, showing strong mixing with the wetting-layer levels, even for small $S$ where the confined electron states are not mixed with wetting-layer states. As indicated later, confined hole states are more localized to the wetting layer than the electron states are and, thus, mix more strongly with wetting-layer states.

The shift of the single-particle band gap [Figs. 3(e) and 3(f)] reflects this dependence on $S$ and on the type of strain.
For biaxially strained dots, the electron and hole levels shift the same way when the bridge is bent and the single-particle gap shift follows the electron and hole shift. For shearing bends, the shift of the electron and hole levels are compensating for small $S$. The band-gap shift follows the hole shift and is negative, because the holes shift more than the electron shift. For large shearing bends both levels mix with the wetting-layer states and a large band-gap reduction occurs.

\section{State distortion and reorientation}

These energy shifts correlate to how the applied mechanical strain shifts electrons and holes inside the dot. Figure 4 shows how the states shift inside the dot as the bridge is bent. The symmetric bend shifts both electrons and holes vertically, either further up into the dot or down toward the wetting layer, as summarized in the schematic. In the flat bridge [Fig. 4(a), $S=0$ ], the electron is more strongly confined to the dot while the hole is close to the wetting layer. When the bridge is biaxially deformed by an upward bend [Fig. 4(a)], both the electron and hole become more localized in the dot and less localized to the wetting layer. For a downward bend, both the electron and hole are pushed toward the wetting layer.

A shearing bend pushes the electrons and holes laterally along the bend. Figures 4(b) and 4(c) show the electron hole probabilities for being in the left and right half of the wetting layer and dot for a shearing bend that pushes up the right half of the dot. Electrons are pushed to the left for small shearing bends, without a large change in the probabilities to be in the dot and wetting layer. For large $S$ the electrons become more localized to the wetting layer and they jump to the right side. Holes, which are more localized to the wetting layer in the unbent structure, shift toward the right to increase the localization in the wetting layer, even for small $S$. For small $S$, the shearing bend laterally separates the electron and hole. However for large $S$ electrons and holes become localized to the same region again.

The applied strain also changes the shape and orientation of the electron and hole. To quantify the thickness of a state in the $z$ direction, we calculate the root mean square (rms) expectation value of $z-z_{\text {ave }}$, where $z_{\text {ave }}$ is the average $z$ position. This gives an average half thickness $T$ :

$$
T=\sqrt{\left\langle\left(z-z_{\mathrm{ave}}\right)^{2}\right\rangle} .
$$

For a symmetrically bent structure, the average in-plane position is just $x_{\text {ave }}=y_{\text {ave }}=0$. We define the average length of a state, $L(\Theta)$, along the in-plane direction given by the in-plane polar angle $\Theta$ to be

$$
L(\Theta)=\sqrt{\left\langle[x \cos (\Theta)+y \sin (\Theta)]^{2}\right\rangle} .
$$

We define the (half) length $L$ of the state to be the maximum of $L(\Theta)$ and the width $W$ of the state to be the minimum $L(\Theta)$. By symmetry, the width is along the axis rotated 90 degrees from the length, The electron and hole are oblong. The orientation of the state is defined to be the direction of maximum length, the $\Theta$ that maximizes $L(\Theta)$.

The dependence of the electron and hole shape and orientation on applied biaxial deformation is shown in Fig. 5. In general, the electron shape and orientation are not strongly sensitive to the applied strain, showing only a few percent 

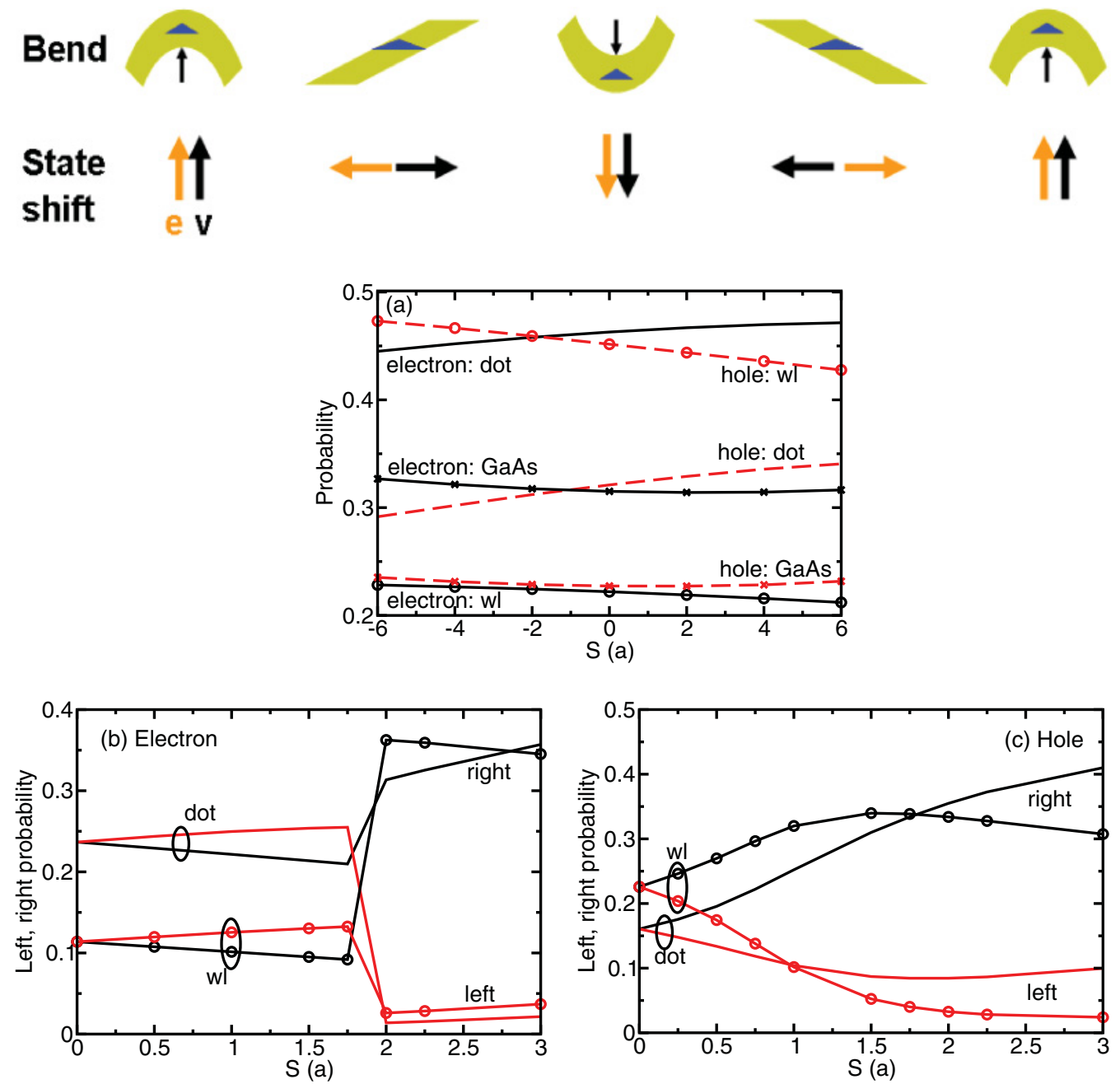

FIG. 4. (Color online) Schematic of the spatial shift of electron (e) and hole (v) position inside a mechanically strained dot. (a) Probability for the lowest electron and hole level to be in the dot, the wetting layer (wl), and the surrounding GaAs barrier region for biaxially deformed dots. Probability for the lowest electron (b) and hole (c) level to be in the left and right halves of the dot and wetting layer for a shearing strain.

change in size and only a few degrees change from the orientation along [110] in an unbent structure. In contrast, the hole shape and orientation are more sensitive to the strain. The hole shows a fifteen percent change in aspect ratio $(L / W)_{h}$, becoming more oblong for $S>0$ and more circular for $S<0$. The orientation of the hole changes dramatically. The hole is oriented along [1 10$]$ in an unbent structure. The hole rotates 30 degrees toward the [010] direction for $S>0$. For $S<0$ the hole rotates in the opposite direction, first through [100], then rapidly to [110] for $S$ where the hole is nearly circular, and then toward [010]. The electron shape and orientation are weakly sensitive to applied strain because the electron is more localized to the dot and more pinned by the confinement provided by the dot. The hole, being more localized in the wetting layer, is not as pinned by the confinement and the applied strain can significantly distort the state.

For a shearing strain, the most important strain-induced effect is the shift in electron and hole position, shown in Fig. 6(a). The shift along [100] confirms the trend shown in Fig. 4. While the hole shifts along $x$, it also shifts along $y$. Initially, it shifts along a line at an angle close to [110] [Fig. 6(c)]. For large shears, it is pushed more along [100]. The electron is pushed along [100] for small $S$ but along [110] for large $S$ when mixing with wetting-layer states is significant. Under a shear, the shape of the electron or hole (i.e., the aspect ratio $L / W$ where $L$ and $W$ are defined relative to the center of the state that is shifted by the shearing strain) does not change much. However the length of the hole state is reduced as it is pushed toward the dot edge and it rotates to be more perpendicular to the dot edge. The electron also undergoes a large rotation when it mixes with wetting-layer states.

To understand the mechanical strain effects on electron and hole levels, one must consider the strain-induced changes in the band-edge profiles.$^{52,61}$ For electrons, that change is primarily via the hydrostatic deformation potential. For holes, there is a shear contribution as well. Here we present the simpler physical picture that can be developed for electrons in a bent structure. Figure 7 sketches the biaxial deformation of the dot due to the relaxation of the lattice mismatch. When the bridge is bent symmetrically, the additional internal relaxation tries 

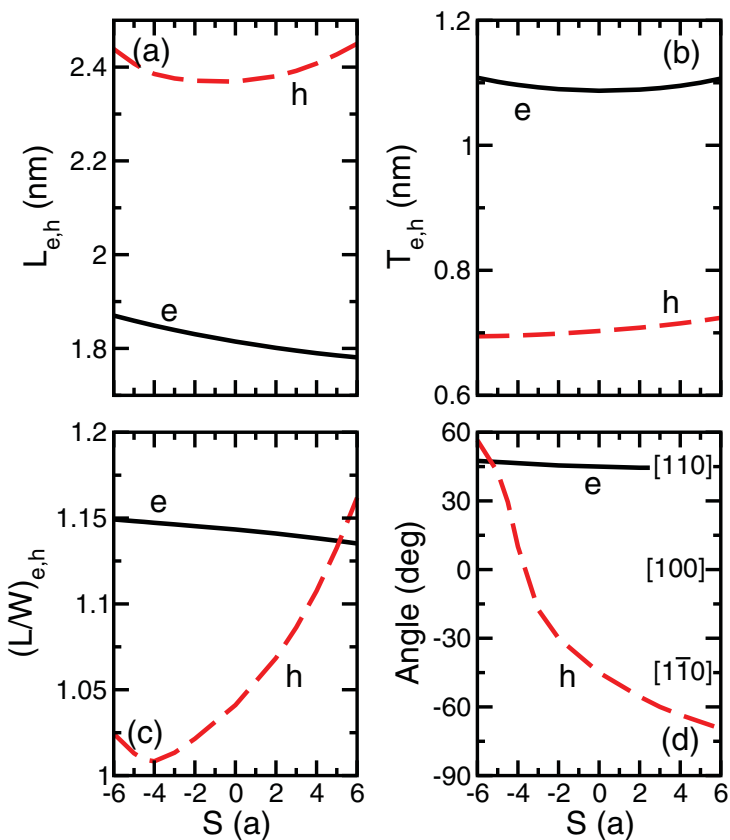

FIG. 5. (Color online) Dependence of the lowest electron (black solid curves) and hole (red dashed curves) shape and orientation on mechanical strain for a biaxially strained dot. (a) The length $L_{e, h}$ of the electron and hole ground state. (b) The thickness $T_{e, h}$ of the electron and hole along $z$. (c) The aspect ratio of the length width (W). (d) In-plane orientation of the electron and hole, defined by the orientation of the long axis. The major crystal directions are indicated.

to undo the bend. The additional relaxation provides vertical expansion (compression) of the QD near the wetting layer and compression (expansion) near the QD apex for $S<0$ $(S>0)$, as indicated in Figs. 7(b) and 7(c). As shown in Fig. 7(d), this relaxation lowers (raises) the conduction band edge in the QD near the wetting layer and raises (lowers) the band edge near the QD apex for $S<0(S>0)$. The effect of the applied strain is a local change in the band profile rather than a spatially uniform shift of the dot band edge. This change in local band profile drives the change in electron position because the electron moves toward the region where the band profile is lowered. It moves more into the dot for $S>0$ and more toward the wetting layer for $S<0$. For $S>0$, the electron moves up into a region of higher band profile. Also the quantum confinement is greater near the apex. Thus the electron energy increases. As $S$ increases further, the electron becomes pinned at the apex. Once the electron is pinned, its energy decreases with increasing $S$ because the band potential continues to decrease with increasing $S$. For $S<0$, the electron moves down into a region of lower potential profile with lower confinement. Both effects lower the electron energy. Over the range shown, no minimum is reached because pinning in the wetting layer is weak. The hole shows the same behavior, except that no maximum is reached for large $S$. The hole does not become pinned in this range of $S$ because it starts off localized closer to the wetting layer in a flat structure.

The effect of a shearing bend on the states is also understood by considering the bend-induced changes in local band profile. Additional strain on the dot due to the shearing bend and the
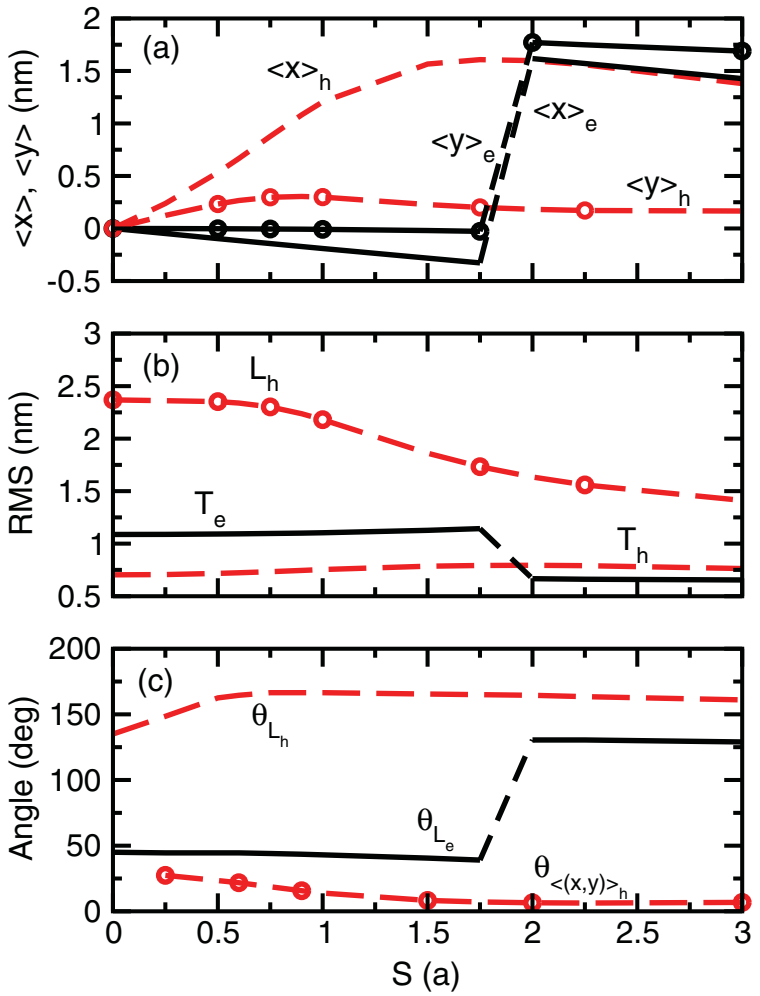

FIG. 6. (Color online) Dependence of the lowest electron (black solid curves) and hole (red dashed curves) position, shape, and orientation on mechanical strain for a shearing strain. (a) The average $x$ and $y$ positions (curves with circles). (b) The length of the hole and thickness for the electron and hole. (c) The orientation of the electron $\left(\Theta_{L_{e}}\right)$ and hole $\left(\Theta_{L_{h}}\right)$ and the angle $\left(\Theta_{\langle(x, y)\rangle_{h}}\right)$ defining the center of the hole state.

relaxation after the bend is compressive. Thus the conduction band edge increases in the dot, pushing the confined electron levels to higher energy, as seen in Fig. 3. When the right side of the dot is pushed up by the shearing strain, the electron levels are initially pushed toward the left side of the dot and down into the wetting layer, as shown in Fig. 4. There is a larger increase in the added compressive strain when moving vertically from the wetting layer into the left side of the dot than when moving into the right side of the dot. This asymmetric change in the local band profile pulls the confined electron level toward the wetting layer and toward the left side. In the wetting layer, the compressive strain is weakened to the left of the dot and enhanced to the right of the dot by a shearing bend. This pulls the wetting-layer states to the left and the lowest wetting-layer states become resonant with the dot states, with increased localization near the right side of the dot to remain orthogonal to the dot states.

\section{Importance of relaxation: linear and nonlinear regimes}

Inclusion of the internal relaxation after the bridge is mechanically deformed is essential for describing the effects of mechanical deformation on the QD. For applied biaxial deformations, calculations (see Fig. 8) done by taking a flat beam with the strain due to lattice mismatch, bending it, but not allowing any additional relaxation, predict energy-level 
(a) Relaxation in a flat bridge

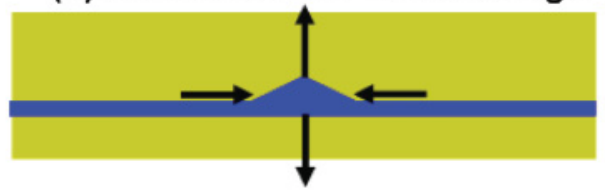

(b) Relaxation in a bent bridge

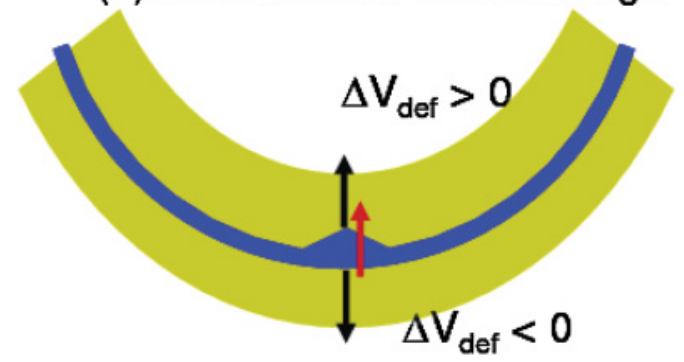

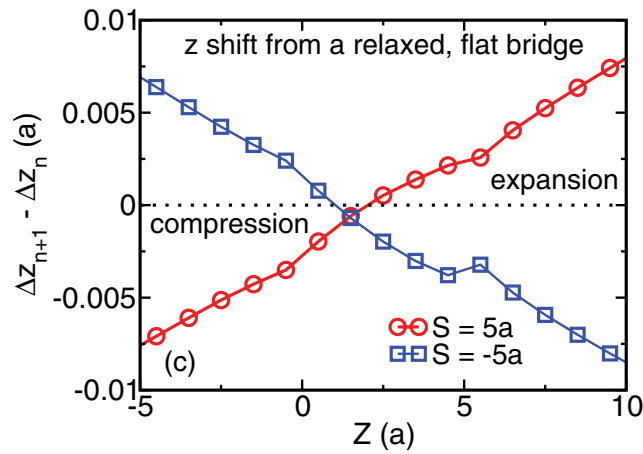

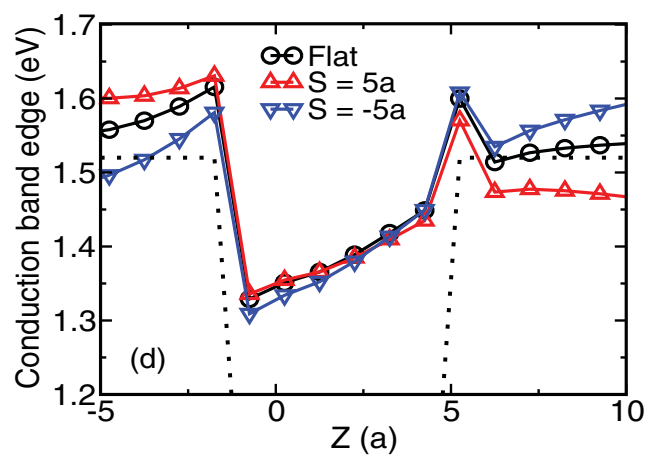

FIG. 7. (Color online) Schematics for (a) lattice relaxation due to lattice mismatch in a flat bridge and (b) the additional relaxation in a bent bridge, (c) the corresponding lattice shift along the vertical axis through the center of the pyramid for biaxial deformations, and (d) the conduction band edge profile along the same vertical axis, for a flat bridge and bent bridges with the indicated amplitudes. The dotted line is the conduction band profile of the dot in a flat bridge before relaxation of the strain due to lattice mismatch.

shifts opposite to those predicted for a fully relaxed structure. The model with partial relaxation also predicts small shifts of electron and hole levels. The results for the model with partial relaxation are both quantitatively and qualitatively different from the results for the fully relaxed structure. For the biaxially deformed bend, the relaxation in response to the bend defines how the electron and hole are repositioned inside the dot and how their energies shift. The electron and hole response to the applied strain is not just a linear response to a small perturbation. The electron and hole response to the external applied strain is nonlinear in that the fully relaxed lattice must be used to model correctly the effect of external strain on the electron and hole levels.

For a shearing bend, the energy levels predicted by the fully relaxed model and the model without relaxation in response to the bend disagree quantitatively but agree qualitatively, as seen in Fig. 8. Both models predict that electrons shift to the left for small $S$. However, the two models predict opposite shifts for holes. Both models predict similar energy shifts for holes because the dominant effect on the hole levels is strain-induced mixing with wetting-layer states, present in both models.

In this regime of nonlinear mechanical response, QD coupling to mechanical modes that is modeled by adding the mechanical deformation as a perturbation to the QD will be suspect unless the mechanical deformation is locally renormalized by the additional relaxation. If the mechanical strains are an order of magnitude weaker, then a linear mechanical response can be adequate. For example, recent experiments have studied the modulation of QD levels by an applied surface acoustic wave (SAW).$^{50}$ The strains on the QD due to a SAW are several orders of magnitude weaker than the strain due to lattice mismatch. Calculations (not presented here) show that internal relaxation in response to the SAW has no effect on the energy-level shifts induced by the SAW. The induced shifts depend linearly on the SAW amplitude and the level shifts follow the same time dependence as the SAW. For the SAW studied, the SAW wavelength was much longer than the dot. The SAW provides a band-edge shift which is nearly uniform across the dot, leading to the energy-level shift that follows the SAW amplitude.

\section{E. Dependence on boundary conditions}

The results shown here apply to a bridge that is shifted vertically and then relaxes with its surface held fixed to maintain the bend. For the biaxially deformed bridge, the major component of the applied strain is along [001]. A shearing bend provides a lateral distortion. There are significant differences in the response for the two cases we have studied. A full understanding of the effects of applied mechanical strain requires that a full range of boundary conditions be studied. ${ }^{16-19,58}$ Dependence on boundary conditions is an opportunity because it means there is flexibility to tailor response by the choice of boundary conditions. Dependence on boundary conditions also presents a challenge if one wants to use QD response to probe local strain, because more information about the QD response will be needed to deconvolve the strain effects that contribute to that response.

\section{F. Coupled dots}

An external biaxial deformation applied to a single QD shifts electrons and holes vertically. A shear strain shifts the 

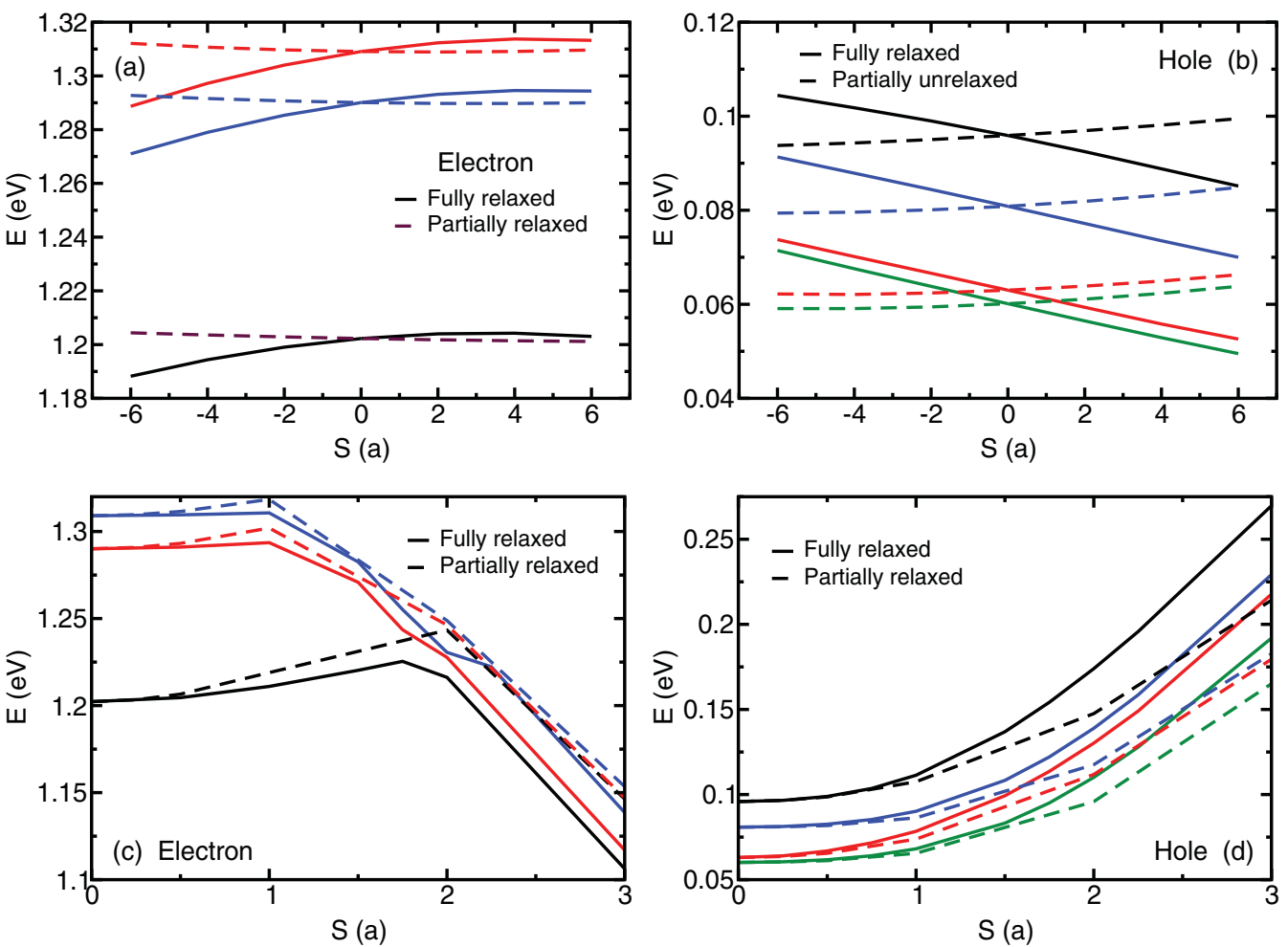

FIG. 8. (Color online) Comparison of electron [(a), (c)] and hole [(b), (d)] levels calculated for a fully relaxed, bent bridge (solid curves) and for a flat bridge strained due to lattice mismatch, then bent but not relaxed further after the mechanical strain is applied (dashed curves), for the biaxially deforming strain [(a), (b)] and the shearing strain [(c), (d)].

electrons and holes laterally. This suggests that applied strain could be used to transfer carriers between dots, much like an electric field does, to control the strength of optical transitions or to interact qubits stored in different dots. To assess these possibilities, we consider both vertically stacked double dots and laterally coupled double dots. We consider a pair of dots consisting of a smaller, pyramidal dot (identical to the dot we have considered so far with base width 12a) and a second dot which is ten percent larger (base width $13 a$ ). For the vertically stacked pair, the dots are aligned with the larger dot on top, as is typical of epitaxially grown dots. We consider a stacked pair with the bottom of the upper wetting layer separated from the apex of the lower dot by $2 a$. For the laterally coupled pair, the dots sit on the same wetting layer separated along [100] (i.e., the direction of the applied strain) with the larger dot located at larger $x$. The lateral separation is taken to be the distance between the interior base edges of the pair.

We consider the same applied strains that were studied for the single dot. For the vertically stacked pair aligned along the same $z$ axis, the applied strains have the same symmetry as when applied to a single dot. For the laterally coupled pair, we take the $y z$ symmetry plane that defines the symmetry of the strain to be the $y z$ plane at the midpoint between the dots.

\section{Vertically stacked double dots}

With the larger QD on top, interdot transfer should be accomplished by vertically shifted electrons and holes down. This is accomplished in single dots with a biaxial deformation with $S<0$. Figure 9(a) shows that the same applied strain leads to an anticrossing of the lowest electron levels from the two dots. Analysis of the electron wave functions confirms that this anticrossing is accompanied by interdot electron transfer. The response of the holes is more complicated, with multiple anticrossings occurring because the hole levels are closer together energetically. The anticrossings are much sharper for holes, because the hole has a heavier mass and is more strongly confined. Electron and hole anticrossings occur at different applied strains. Thus electrons and holes can be transferred separately between dots. The dependence of electron levels on $S$ is similar for the single dot and the stacked pair. For holes, there are bigger differences, indicating that the effects of local strain due to lattice mismatch are modified when the two dots are close together.

A shearing strain laterally shifts electrons and holes and should not be as efficient in transferring charge between vertically stacked dots. We do not find any vertical interdot electron transfer for shearing strains in the range considered so far. For holes, there are some weak anticrossings, especially when the dot states are mixed with the wetting-layer states and among the closely spaced higher levels.

\section{Laterally coupled dots: effect of strain in a flat structure}

Before considering bent structures, it is important to understand the effects of strain due to lattice mismatch on two closely spaced, laterally coupled dots in a flat bridge. Figures 10(a) and 10(b) show how electron and hole levels shift as the interdot separation is reduced. The lowest pair of states arise from the ground states of each dot. As the dot separation is reduced and the states mix, there should be an anticrossing that pushes one state to lower energy and the 

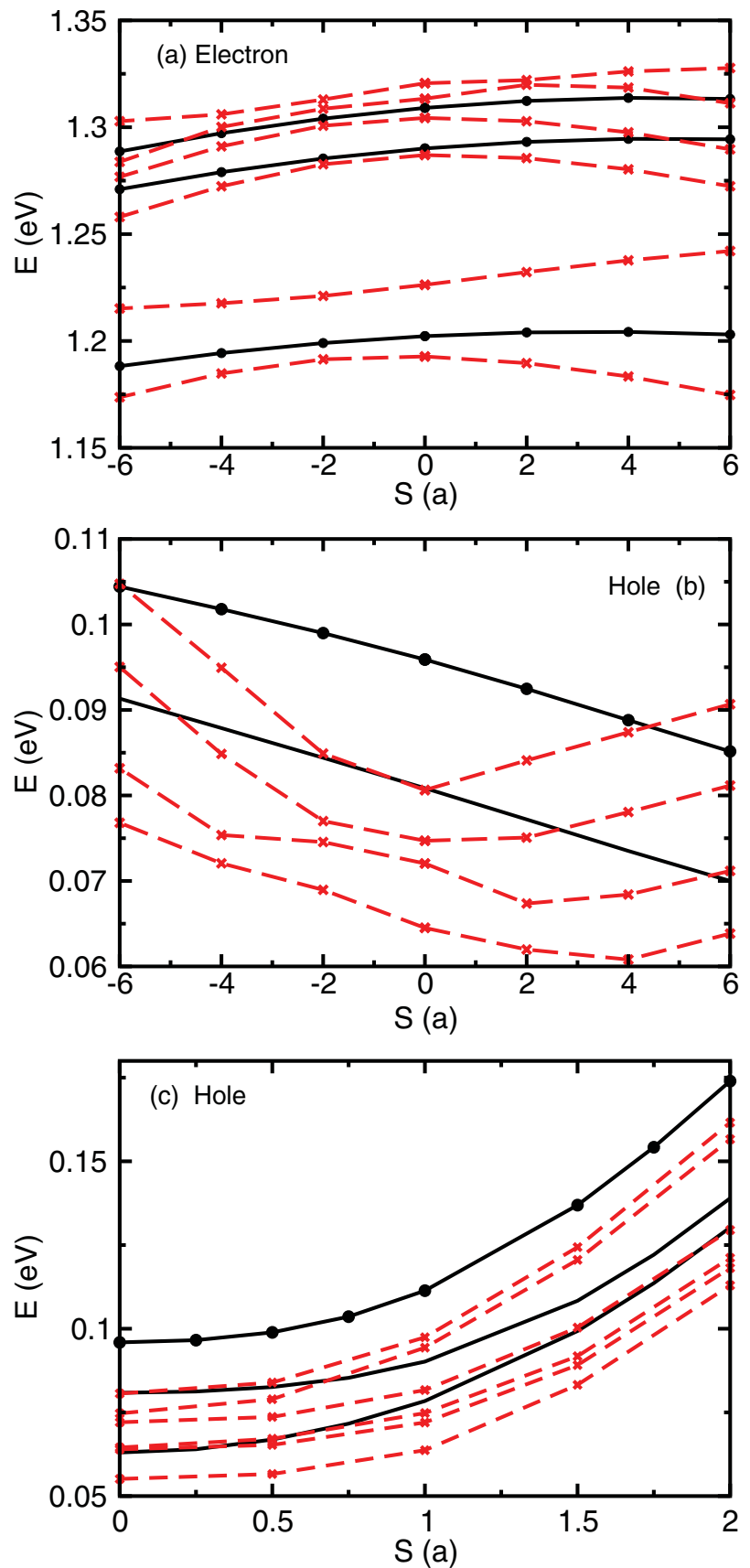

FIG. 9. (Color online) Dependence of the near-band-gap energy levels of a vertically stacked pair of dots (red, dashed curves) on mechanical strain: (a) electron and (b) hole levels in a biaxially deformed bridge, and (c) hole levels for a shearing strain. For comparison, the energy levels of the smaller, bottom dot are shown (black, solid curves). The apex of the bottom dot is separated from the wetting layer of the larger top dot by $2 a$.

other to higher energy. For both electrons and holes, the two states split, as expected, but they also shift farther into the gap, indicating that strain between the dots due to lattice mismatch can significantly reduce the energy levels for closely space dots. The effects are larger for holes, which are more strongly coupled to the wetting-layer states and can tunnel laterally more easily than electrons.
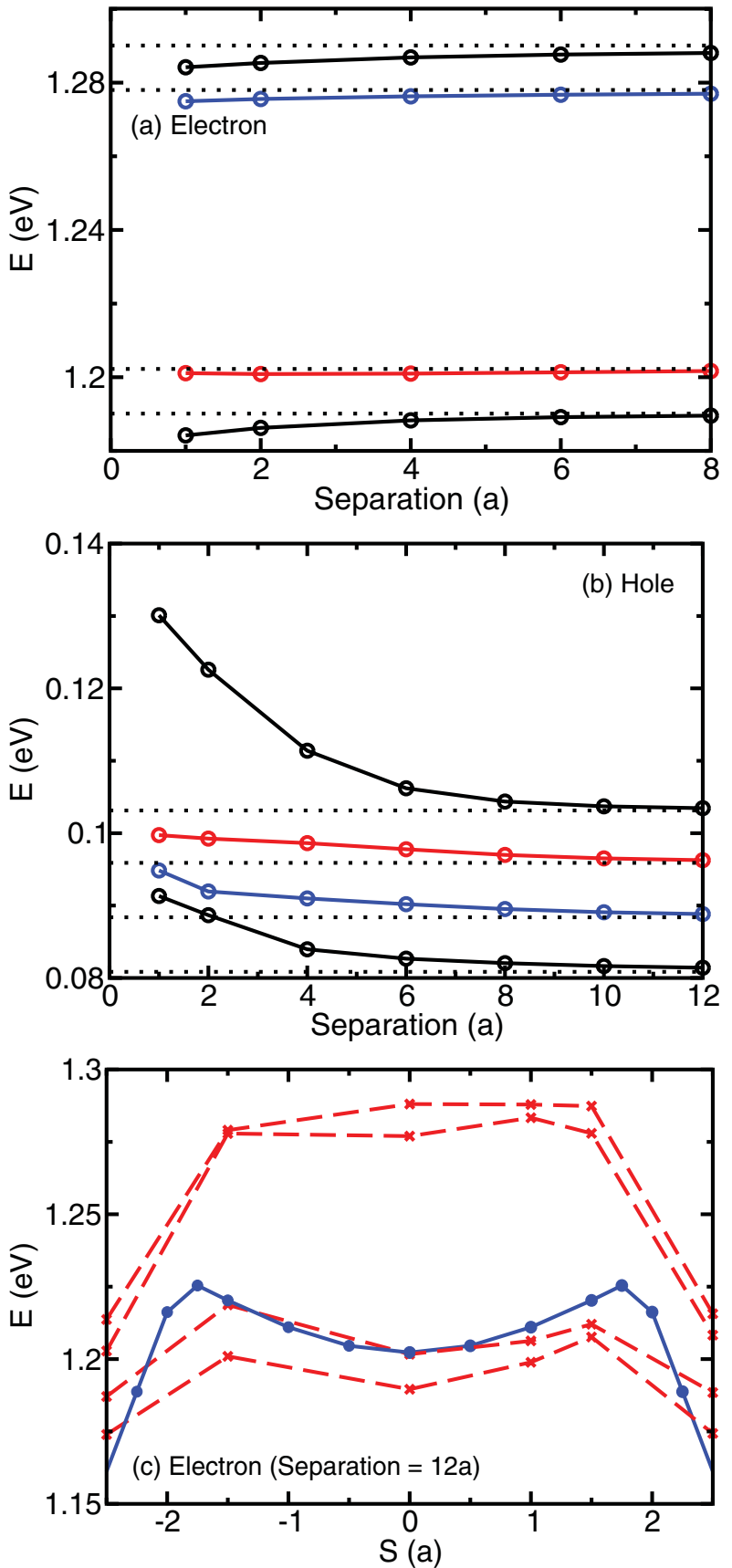

FIG. 10. (Color online) Near-band-gap energy levels of a laterally coupled dot pair: the dependence of the energy levels on interdot edge-to-edge separation for electrons (a) and holes (b) in a dot pair in a flat bridge. Energies of the coupled dot pair are shown as solid lines. Energy levels for the two isolated dots are shown as dotted curves. (c) Dependence of the electron levels (red, dashed curves) in a dot pair on applied shearing strain. The dot-to-dot separation is $12 a$. The lowest electron level in the small dot is shown (solid curve).

\section{Laterally coupled dots: effect of strain in a bent structure}

Interdot strain due to lattice mismatch couples and splits the two lowest levels, so interdot strain inhibits interdot tunneling. To consider the effects of applied strain, we have studied widely spaced dots with separation $12 a$ where the effects of interdot strain are less important. A shearing strain applied to a single dot shifts the electrons laterally. As shown in Fig. 10(c), 
a level anticrossing does occur for the shear $(S>0)$ that should push electrons laterally from the larger dot toward the smaller dot. This interdot transfer is inhibited if the separation is reduced. Biaxial deformations, which provide vertical transfer, do not induce lateral interdot transfer. Interdot lateral transfer of holes is also possible with a shear strain, but not with a biaxial deformation.

\section{G. Excitons}

\section{Binding energy and fine structure}

The lowest electron and hole states are doubly degenerate due to spin. Consequently, the lowest electron-hole pair state is fourfold degenerate. When the Coulomb and exchange effects are included, the pair ground state splits into four exciton states. Two are dark excitons (DEs) and two are bright excitons (BEs). Figures 11(a) and 11(b) compare the strain-induced change in energy of the four lowest mechanoexciton states and the lowest electron-hole pair state for (a) biaxial deformation and (b) shearing strain. On a gross scale, the strain-induced change in exciton levels follows the strain-induced change of the pair ground state, with the change in pair energy making the biggest contribution to the change in exciton energy. On a finer scale, the effect of applied strain on the Coulomb and exchange energies is more dramatic, as seen in the exciton fine structure shown in Figs. 11(c) and 11(d) and Figs. 13(a) and 13(b). DEs are nearly degenerate and split from the pair ground state by the binding energy. The binding energy increases (decreases) when a biaxial deformation is applied with $S>0$ $(S<0)$ because the electron and hole are pushed more into (out of) the QD. The binding increases when a shearing bend is applied, even though the electron and hole are pushed laterally in opposite directions, because the vertical electron-hole separation is reduced by the shearing bend. The two BEs are split from the DEs by the isotropic exchange interaction and the two BEs are further split by the anisotropic contribution to the exchange coupling. The binding energy and the DE-BE exchange splitting change slowly when the nanomechanical strain is applied [see Figs. 13(a) and 13(b)]. However, nanomechanical strain can significantly alter the AES, with reduced splitting and an apparent anticrossing for a biaxial deformation when $S>0$ and greatly increased AES from an apparent level repulsion for a shearing bend. As will be discussed later, these apparent level anticrossings and repulsion actually arise from strain-induced changes in the anisotropic exchange coupling rather than from avoided level crossings.

\section{Bright-exciton polarization}

When the mechanical strain is applied, the dark excitons remain dark and the bright excitons remain bright. The mechanical strain does not strongly mix the spin-forbidden transitions with change in total $z$ angular momentum $\Delta J_{z} \neq \pm 1$, which define the DEs, with the spin-allowed transitions with $\Delta J_{z}= \pm 1$, which define the BEs. However, the polarization of the BEs is drastically rotated by the applied strain. The two BEs are orthogonally polarized. In a flat bridge they are polarized along the QD diagonals, as shown in Fig. 12. For the largest bends considered in a biaxially deformed bridge, the lowest $\mathrm{BE}$ is polarized along the [100] bend direction for $S>0$ and approaches this limit for $S<0$. For a shearing bend, the polarization of the lowest BE rotates
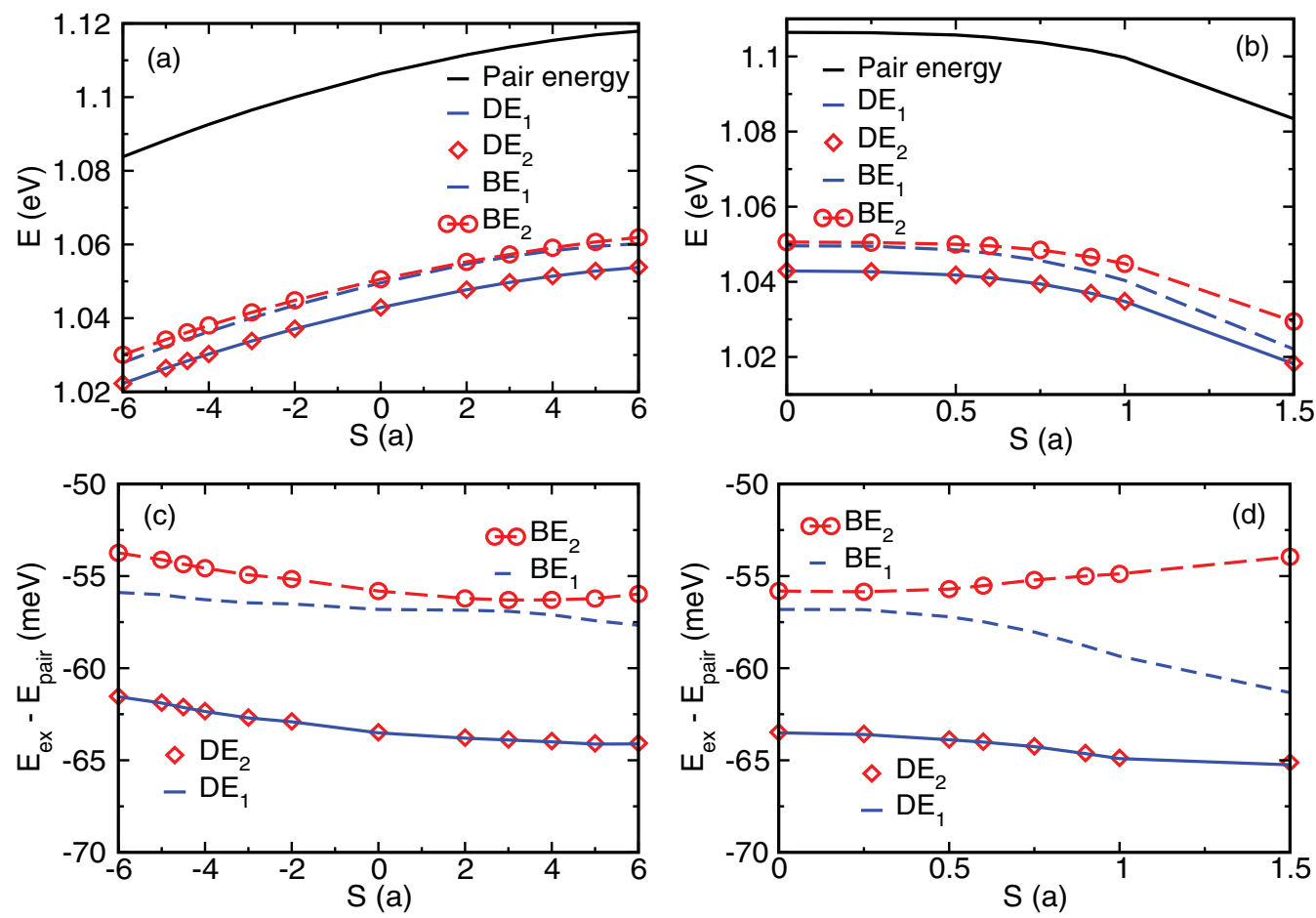

FIG. 11. (Color online) The energies of the lowest two bright excitons (BE) and two dark excitons (DE) and the single-particle pair energy for (a) biaxial deformation and (b) a shearing bend. Dependence of the fine structure for the lowest two bright excitons (BE) and two dark excitons (DE) on bend amplitude for (c) biaxial deformation and (d) a shearing bend. 

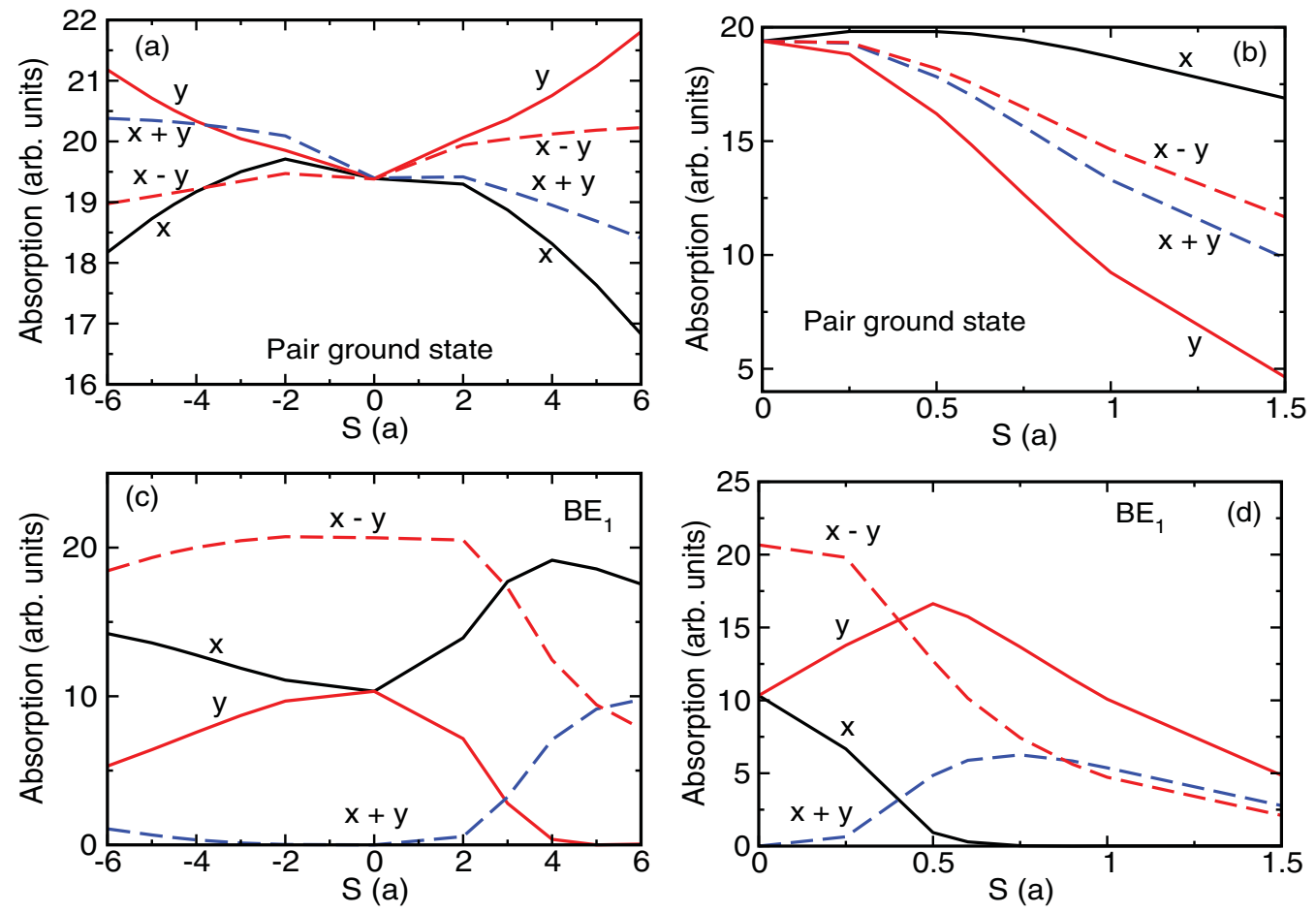

FIG. 12. (Color online) Dependence of the absorption strength of the single-particle pair ground state on the bend amplitude of (a) a biaxial deformation and (b) a shearing bend for polarization along the pyramid diagonals $(x+y$ and $x-y)$ and along the pyramid sides parallel ( $x$ ) and perpendicular $(y)$ to the bend. Dependence of the absorption strength of the lowest BE on the bend amplitude of (c) a biaxial deformation and (d) a shearing bend.

in the opposite direction, becoming polarized perpendicular to the bend when $S \simeq 2 a$ and the electron states cross the wetting-layer states. The two BEs remain orthogonally polarized for each strain and rotate together.

The absorption strength for the pair ground state is shown in Fig. 12. In Figs. 12(a) and 12(b), we sum over the possible transitions to the four degenerate pair ground states. In a flat structure, the average absorption into the pair state has no polarization dependence. When a biaxial strain is applied, the pair ground state transition is enhanced by ten percent for polarization along $y$, is suppressed by ten percent for polarization along $x$, and less affected for polarization along the diagonals. This weak dependence of the absorption of the pair ground state on strain contrasts with the effect of applied strain on bright excitons [Figs. 12(c) and 12(d)], which show the large polarization rotation and 100 percent changes in polarization.

The absorption strength of the pair ground state is determined by the magnitude of the pair dipole matrix elements. The weak dependence on strain is an another measure of the effect of strain on the electron-hole overlap. For a biaxial deformation, the effect is weak because the electron and hole shift in the same direction under the applied strain.

For a shearing bend, the pair ground state absorption shows a stronger dependence on strain, with a suppression of the absorption for larger $S$. This suppression arises because the electron and hole are shifted laterally in opposite directions by a shearing strain, making the pair transition spatially less direct. This is an effect of the strain on the dipole matrix element. The BE absorption strength is also suppressed at larger $S$ due to these matrix-element effects. However, the large rotation of the $\mathrm{BE}$ polarization is still clear. The pair ground state is not as strongly polarized by the strain and does not show a large rotation. Again, the strong sensitivity of the BE polarization arises from the strain-induced changes to the mixing.

\section{Understanding the effect of strain on exchange coupling, fine-structure splitting, and polarization}

For the QDs considered here, the two lowest BE states are made primarily from the lowest electron-hole pair state, with minimal mixing of higher confined states. Thus the BEs are mixtures of the two degenerate, $J_{z}= \pm 1$ electron-hole pair states excited optically by a spin-conserving transition with circularly polarized light $\left(\Delta J_{z}= \pm 1\right)$. In this basis of pair states, the Hamiltonian for the two BEs is

$$
H_{\mathrm{BE}}=\left(\begin{array}{lr}
E_{\text {eh }}+V_{\text {coul }}+V_{\text {exch,sc }} & V_{\text {exch,mix }} \\
V_{\text {exch,mix }}^{*} & E_{\text {eh }}+V_{\text {coul }}+V_{\text {exch,sc }}
\end{array}\right) .
$$

$E_{\text {eh }}$ is the electron-hole pair energy. The (real) Coulomb matrix element $V_{\text {coul }}$ determines the binding energy but does not mix the spin states with different $J_{z}$. The exchange splitting between DEs and BEs is determined by a (real) exchange matrix element which is also spin conserving (sc), $V_{\text {exch,sc }}$. The AES between BEs is determined by the magnitude of the (complex) off-diagonal exchange interaction $V_{\text {exch,mix }}$ which does mix $J_{z} \cdot V_{\text {coul }}$ and $V_{\text {exch,sc }}$ are much larger than $\left|V_{\text {exch,mix }}\right|$, but strain-induced changes in $V_{\text {coul }}$ and $V_{\text {exch,sc }}$ are comparable to $\left|V_{\text {exch,mix }}\right|$, as shown in Fig. 13. This is true for biaxial deforming bends, shown in Fig. 13, and shearing bends (not shown). $\Delta V_{\text {coul }}, \Delta V_{\text {exch,sc }}$, and $\left|V_{\text {exch,mix }}\right|$ follow, respectively, 

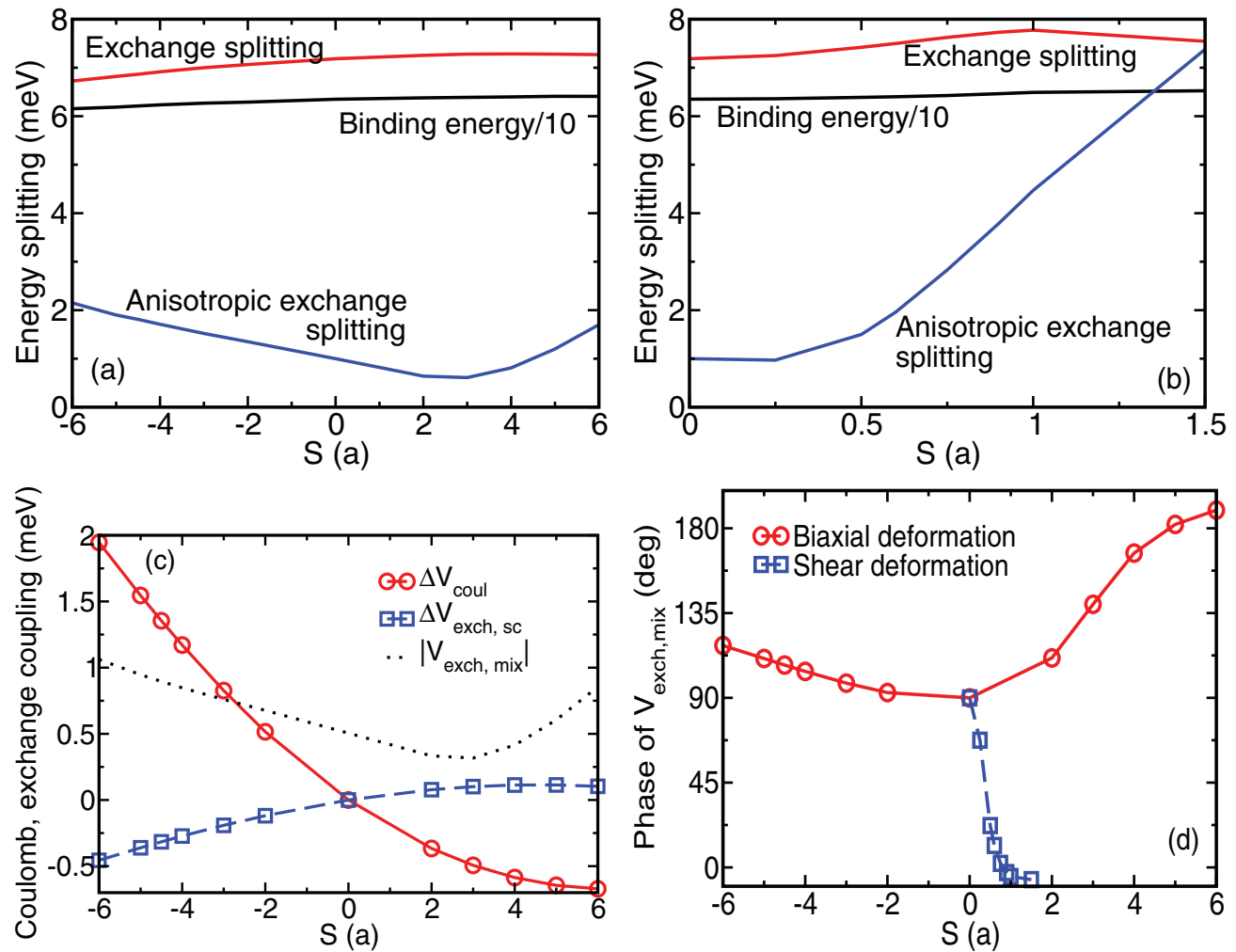

FIG. 13. (Color online) Exciton energy splittings for (a) a biaxial deformation and (b) a shearing bend. (c) Dependence of the strain-induced change in the (real) Coulomb coupling $\Delta V_{\text {coul }}$ and spin-conserving exchange coupling $\Delta V_{\text {exch,sc }}$ and the magnitude of the (complex) exchange coupling that mixes spin $\left|V_{\text {exch,mix }}\right|$ on the bend amplitude for a biaxial deformation. (d) Dependence of the phase of the exchange coupling $V_{\text {exch,mix }}$ for spin mixing on bend amplitude.

the change in binding energy, the DE/BE exchange splitting, and the AES shown in Figs. 13(a) and 13(b) for biaxially deforming bends and shearing bends. $V_{\text {coul }}$ and $V_{\text {exch,sc }}$ vary smoothly under strain while $\left|V_{\text {exch,mix }}\right|$ shows the apparent anticrossing for the biaxial deforming bend and the apparent level repulsion for the shearing bend. This is a matrix-element effect rather than a true anticrossing, because the exciton character does not switch near these apparent anticrossings.

Only the two optically active $J_{z}= \pm 1$ pair states are mixed to make the $\mathrm{BE}$, so the phase of the mixing is determined by the phase of $V_{\text {exch,mix }}$, which is shown in Fig. 13. In turn, the polarization of the optical transition is determined by the phase of the mixing, as shown schematically in Fig. 14. When the phases for the two optically active pair states are chosen so that their optical dipole moments are proportional to $\hat{x} \pm i \hat{y}$, then $V_{\text {exch,mix }}$ is imaginary for $S=0$, ensuring that BEs are polarized along the QD diagonals in a flat bridge. When strain is applied, the phase of $V_{\text {exch,mix }}$ and the polarization rotate. When $V_{\text {exch,mix }}$ is real, the mixing leads to a polarization either along $x$ or $y$. The sign of $V_{\text {exch,mix }}$ determines whether the polarization rotates by $\pi / 4$ to $x$ or $-\pi / 4$ to $y$.

The reason why strain controls the phase of $V_{\text {exch,mix }}$ emerges by looking at Coulomb and exchange matrix elements. $V_{\text {coul }}$ couples the electron density at $\mathbf{r}\left[\rho_{e}(\mathbf{r})=\left|\phi_{e}(\mathbf{r})\right|^{2}\right.$, for electron state $\left.\phi_{e}(\mathbf{r})\right]$ with hole density at $\mathbf{r}^{\prime}\left[\rho_{h}\left(\mathbf{r}^{\prime}\right)=\right.$ $\left.\left|\phi_{h}\left(\mathbf{r}^{\prime}\right)\right|^{2}\right]$ :

$$
V_{\text {coul }}=-\int \frac{\rho_{e}(\mathbf{r}) \rho_{h}\left(\mathbf{r}^{\prime}\right)}{\epsilon\left(\mathbf{r}, \mathbf{r}^{\prime}\right)\left|\mathbf{r}-\mathbf{r}^{\prime}\right|},
$$

with the local screening given by $\epsilon\left(\mathbf{r}, \mathbf{r}^{\prime}\right) . \quad V_{\text {coul }}$ is real and, from the Kramers degeneracy of the spin states, spin
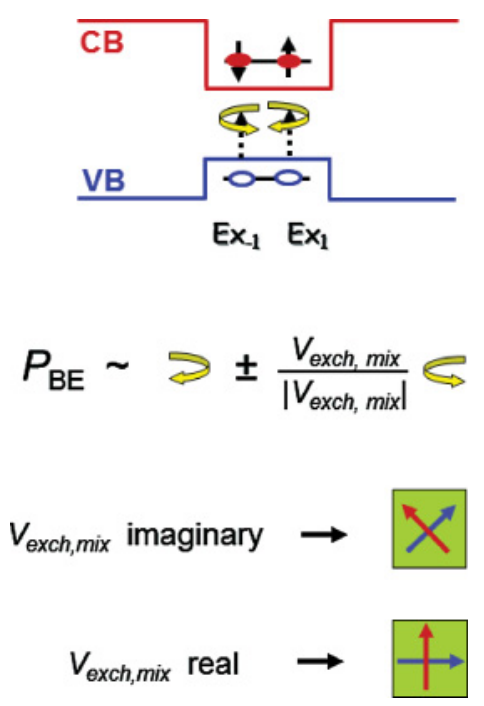

FIG. 14. (Color online) Schematic for the mixing of the two allowed, circularly polarized transitions (top line) that lead to the bright excitons. The connection between the anisotropic exchange coupling and the resulting polarization $P_{\mathrm{BE}}$ is indicated (second line). The phase of $V_{\text {exch,mix }}$ determines the polarization of the BEs [arrows inside the QD base (third and fourth line)] made by mixing the circularly polarized pair transitions. 
independent. $V_{\text {exch,sc }}$ couples polarization density $P(\mathbf{r})$ at $\mathbf{r}$, with $P(\mathbf{r})=\phi_{e}(\mathbf{r}) \phi_{h}(\mathbf{r})$, and the conjugate polarization density at $\mathbf{r}^{\prime}, P^{*}\left(\mathbf{r}^{\prime}\right)$ :

$$
V_{\mathrm{exch}, \mathrm{sc}}=\int \frac{P(\mathbf{r}) P^{*}\left(\mathbf{r}^{\prime}\right)}{\epsilon\left(\mathbf{r}, \mathbf{r}^{\prime}\right)\left|\mathbf{r}-\mathbf{r}^{\prime}\right|} .
$$

$V_{\text {exch,sc }}$ is real, depends mostly on the interaction weighted average of $|P(\mathbf{r})|\left|P\left(\mathbf{r}^{\prime}\right)\right|$ and weakly on the phase difference between $P(\mathbf{r})$ and $P\left(\mathbf{r}^{\prime}\right)$. For $V_{\text {exch,mix }}$, the Kramers degeneracy of pairs with opposite spin ensures that the coupling is between $P(\mathbf{r})$ and $P\left(\mathbf{r}^{\prime}\right)$ :

$$
V_{\text {exch,mix }}=\int \frac{P(\mathbf{r}) P\left(\mathbf{r}^{\prime}\right)}{\epsilon\left(\mathbf{r}, \mathbf{r}^{\prime}\right)\left|\mathbf{r}-\mathbf{r}^{\prime}\right|} .
$$

$V_{\text {exch,mix }}$ and $V_{\text {exch,sc }}$ depend similarly on $|P(\mathbf{r})|$, but $V_{\text {exch,mix }}$ depends on the sum of the phases of $P(\mathbf{r})$ and $P\left(\mathbf{r}^{\prime}\right)$. Mechanical strain modifies binding energy and DE-BE exchange splitting by reshaping the electron and hole densities. The smooth variation of binding energy and exchange splitting reflect limited strain-induced reshaping. The exciton polarization and phase of spin mixing are modified by changing the relative phase and orientation of the electron and hole pair.

Recently it was found that BE polarization of an alloy quantum dot can vary significantly for QDs with the same composition profile but different actual atom distributions even though energy splittings were not as sensitive to the distribution. ${ }^{59}$ This is another indication of the tight connection between polarization, the phase of spin mixing, and the relative orientation of electrons and holes. Experimentally a strong connection between the sign of the asymmetric exchange coupling, the size of the fine-structure splitting, and the orientation of polarization has been observed for quantum dots manipulated by electric field ${ }^{9}$ and strain. ${ }^{18}$

\section{SUMMARY}

In conclusion, we have used atomistic tight-binding theory to show how nanomechanical strain can be used to dynamically control the optics of quantum dots. Electron and hole levels and charge distributions can shift together or in opposite directions depending on how the strain is applied. This gives control to tailor optical properties. The strain can also be used to transfer electrons and holes between coupled dots to control transition strengths and interacting qubits for quantum-information processing. The internal strain due to the lattice mismatch, the nanomechanical strain, and the internal readjustment to minimize the applied strain must all be accounted for to model correctly the strain effects. Applied strain can be used to manipulate the fine-structure splitting of mechanoexcitons by reshaping and reorienting the electron and hole charge distributions. Importantly, strain can be used to control the phase of the spin mixing and rotate the polarization of mechanoexcitons, giving control to reengineer the inner workings of the exciton.

\section{ACKNOWLEDGMENTS}

M.Z. would like to acknowledge the support by the Foundation for Polish Science, Homing Plus Programme cofinanced by the European Union within the European Regional Development Fund. *garnett.bryant@nist.gov

${ }^{1}$ O. Benson, C. Santori, M. Pelton, and Y. Yamamoto, Phys. Rev. Lett. 84, 2513 (2000)

${ }^{2}$ N. Akopian, N. H. Lindner, E. Poem, Y. Berlatzky, J. Avron, D. Gershoni, B. D. Gerardot, and P. M. Petroff, Phys. Rev. Lett. 96, 130501 (2006).

${ }^{3}$ A. Dousse, J. Suffczyński, A. Beveratos, O. Krebs, A. Lemaïtre, I. Sagnes, J. Bloch, P. Voisin, and P. Senellart, Nature (London) 466, 217 (2010).

${ }^{4}$ R. J. Young, R. M. Stevenson, A. J. Shields, P. Atkinson, K. Cooper, D. A. Ritchie, K. M. Groom, A. I. Tartakovskii, and M. S. Skolnick, Phys. Rev. B 72, 113305 (2005).

${ }^{5}$ R. M. Stevenson, R. J. Young, P. See, D. G. Gevaux, K. Cooper, P. Atkinson, I. Farrer, D. A. Ritchie, and A. J. Shields, Phys. Rev. B 73, 033306 (2006).

${ }^{6}$ K. Kowalik, O. Krebs, A. Lemaitre, S. Laurent, P. Senellart, P. Voisin, and J. A. Gaj, Appl. Phys. Lett. 86, 041907 (2005).

${ }^{7}$ B. G. Gerardot, S. Seidl, P. A. Dalgarno, R. J. Warburton, D. Granados, J. M. Garcia, K. Kowalik, and O. Krebs, Appl. Phys. Lett. 90, 041101 (2007).

${ }^{8}$ M. E. Reimer, M. Korkusinski, D. Dalacu, J. Lefebvre, J. Lapointe, P. J. Poole, G. C. Aers, W. R. McKinnon, P. Hawrylak, and R. L. Williams, Phys. Rev. B 78, 195301 (2008).
${ }^{9}$ A. J. Bennett, M. A. Pooley, R. M. Stevenson, M. B. Ward, R. B. Patel, A. Boyer de la Giroday, N. Sköld, I. Farrer, C. A. Nicoll, D. A. Ritchie, and A. J. Shields, Nature Phys. 6, 947 (2010).

${ }^{10}$ J. D. Mar, X. L. Xu, J. S. Sandhu, A. C. Irvine, M. Hopkinson, and D. A. Williams, Appl. Phys. Lett. 97, 221108 (2010).

${ }^{11}$ A. Muller, W. Fang, J. Lawall, and G. S. Solomon, Phys. Rev. Lett. 101, 027401 (2008).

${ }^{12}$ A. Muller, W. Fang, J. Lawall, and G. S. Solomon, Phys. Rev. Lett. 103, 217402 (2009).

${ }^{13}$ T. Nakaoka, T. Kakitsuka, T. Saito, S. Kako, S. Ishida, M. Nisioka, Y. Yoshikuni, and Y. Arakawa, J. Appl. Phys. 94, 6812 (2003).

${ }^{14}$ T. Nakaoka, T. Kakitsuka, T. Saito, and Y. Arakawa, Appl. Phys. Lett. 84, 1392 (2004).

${ }^{15}$ J. R. Gell, M. B. Ward, R. J. Young, R. M. Stevenson, P. Atkinson, D. Anderson, G. A. C. Jones, D. A. Ritchie, and A. J. Shields, Appl. Phys. Lett. 93, 081115 (2008).

${ }^{16}$ S. Seidl, M. Kroner, A. Hogele, K. Karrai, R. J. Warburton, B. D. Gerardot, and P. M. Petroff, Appl. Phys. Lett. 88, 203113 (2006).

${ }^{17}$ F. Ding, R. Singh, J. D. Plumhof, T. Zander, V. Křápek, Y. H. Chen, M. Benyoucef, V. Zwiller, K. Dörr, G. Bester, A. Rastelli, and O. G. Schmidt, Phys. Rev. Lett. 104, 067405 (2010).

${ }^{18}$ J. D. Plumhof, V. Křápek, F. Ding, K. D. Jöns, R. Hafenbrak, P. Klenovský, A. Herklotz, K. Dörr, P. Michler, A. Rastelli, and O. G. Schmidt, Phys. Rev. B 83, 121302 (2011). 
${ }^{19}$ R. Singh and G. Bester, Phys. Rev. Lett. 104, 196803 (2010).

${ }^{20}$ G. W. Bryant, M. Zieliński, N. Malkova, J. Sims, W. Jaskólski, and J. Aizpurua, Phys. Rev. Lett. 105, 067404 (2010).

${ }^{21} \mathrm{M}$. Eichenfield, R. Camacho, J. Chan, K. J. Vahala, and O. Painter, Nature (London) 459, 550 (2009).

${ }^{22}$ J. Rosenberg, Q. Lin, and O. Painter, Nature Photonics 3, 478 (2009).

${ }^{23}$ T. Zander, A. Herklotz, S. Kiravittaya, M. Benyoucef, F. Ding, P. Atkinson, S. Kumar, J. D. Plumhof, K. Dörr, A. Rastelli, and O. G. Schmidt, Opt. Express 17, 22452 (2009).

${ }^{24}$ V. Prinz, V. Seleznev, A. Gutakovsky, A. Chehovskiy, V. Preobrazhenskii, M. Putyato, and T. Gavrilona, Physica E 6, 828 (2000).

${ }^{25}$ T. Kipp, H. Welsch, Ch. Strelow, Ch. Heyn, and D. Heitmann, Phys. Rev. Lett. 96, 077403 (2006).

${ }^{26}$ S. Mendach, R. Songmuang, S. Kiravittaya, A. Rastelli, M. Benyoucef, and O. Schmidt, Appl. Phys. Lett. 88, 111120 (2006).

${ }^{27}$ X. Li, J. Phys. D 41, 193001 (2008).

${ }^{28}$ S. Vicknesh, F. Li, and Z. Mi, Appl. Phys. Lett. 94, 081101 (2009).

${ }^{29}$ K. L. Ekinci, Y. T. Yang, and M. L. Roukes, J. Appl. Phys. 95, 2682 (2004).

${ }^{30}$ Y. T. Yang, C. Callegari, X. L. Feng, K. L. Ekinci, and M. L. Roukes, Nano Lett. 6, 583 (2006).

${ }^{31}$ K. Jensen, K. Kim, and A. Zettl, Nature Nanotech. 3, 533 (2008).

${ }^{32}$ R. H. Blick, H. Qin, H.-S. Kim, and R. A. Marsland, New J. Phys. 9, 241 (2007).

${ }^{33}$ I. Mahboob and H. Yamaguchi, Nature Nanotech. 3, 275 (2008).

${ }^{34}$ A. A. Kovalev, G. E. W. Bauer, and A. Brataas, Phys. Rev. Lett. 94, 167201 (2005).

${ }^{35}$ M. S. Majdoub, P. Sharma, and T. Çağin, Phys. Rev. B 78, 121407 (2008).

${ }^{36}$ K. C. Schwab, E. A. Hendriksen, J. M. Worlock, and M. L. Roukes, Nature (London) 404, 974 (2000).

${ }^{37}$ M. D. LaHaye, O. Buu, B. Camarota, and K. C. Schwab, Science 304, 74 (2004).

${ }^{38}$ R. G. Knobel and A. N. Cleland, Nature (London) 424, 291 (2003).

${ }^{39}$ I. Wilson-Rae, P. Zoller, and A. Imamoglu, Phys. Rev. Lett. 92, 075507 (2004).

${ }^{40}$ K. Hammerer, M. Aspelmeyer, E. S. Polzik, and P. Zoller, Phys. Rev. Lett. 102, 020501 (2009).
${ }^{41}$ C. Rocke, S. Zimmermann, A. Wixforth, J. P. Kotthaus, G. Bohm, and G. Weimann, Phys. Rev. Lett. 78, 4099 (1997).

${ }^{42}$ A. O. Govorov, A. V. Kalameitsev, V. M. Kovalev, H.-J. Kutschera, and A. Wixforth, Phys. Rev. Lett. 87, 226803 (2001).

${ }^{43}$ F. Alsina, J. A. H. Stotz, R. Hey, and P. V. Santos, Solid. State. Commun. 129, 453 (2003).

${ }^{44}$ M. M. de Lima Jr. and P. V. Santos, Rep. Prog. Phys. 68, 1639 (2005).

${ }^{45}$ W. J. M. Naber, T. Fujisawa, H. W. Liu, and W. G. van der Wiel, Phys. Rev. Lett. 96, 136807 (2006).

${ }^{46}$ M. Kataoka, R. J. Schneble, A. L. Thorn, C. H. W. Barnes, C. J. B. Ford, D. Anderson, G. A. C. Jones, I. Farrer, D. A. Ritchie, and M. Pepper, Phys. Rev. Lett. 98, 046801 (2007).

${ }^{47}$ M. R. Astley, M. Kataoka, C. J. B. Ford, C. H. W. Barnes, D. Anderson, G. A. C. Jones, I. Farrer, D. A. Ritchie, and M. Pepper, Phys. Rev. Lett. 99, 156802 (2007).

${ }^{48}$ T. Sogawa, H. Sanada, H. Gotoh, H. Yamaguchi, S. Miyashita, and P. V. Santos, Phys. Rev. B 80, 075304 (2009).

${ }^{49}$ O. D. D. Couto Jr., S. Lazić, F. Iikawa, J. A. H. Stotz, U. Jahn, R. Hey, and P. V. Santos, Nature Photonics 3, 645 (2009).

${ }^{50}$ M. Metcalfe, S. M. Carr, A. Muller, G. S. Solomon, and J. Lawall, Phys. Rev. Lett. 105, 037401 (2010).

${ }^{51}$ J. G. Diaz, G. W. Bryant, W. Jaskólski, and M. Zieliński, Phys. Rev. B 75, 245433 (2007).

${ }^{52}$ W. Jaskólski, M. Zieliński, G. W. Bryant, and J. Aizpurua, Phys. Rev. B 74, 195339 (2006).

${ }^{53}$ J. G. Diaz, M. Zieliński, W. Jaskólski, and G. W. Bryant, Phys. Rev. B 74, 205309 (2006).

${ }^{54}$ K. Leung and K. B. Whaley, Phys. Rev. B 56, 7455 (1997).

${ }^{55}$ A. Franceschetti, H. Fu, L. W. Wang, and A. Zunger, Phys. Rev. B 60, 1819 (1999).

${ }^{56}$ S. Lee, L. Jönsson, J. W. Wilkins, G. W. Bryant, and G. Klimeck, Phys. Rev. B 63, 195318 (2001).

${ }^{57}$ G. Bester, S. Nair, and A. Zunger, Phys. Rev. B 67, 161306 (2003)

${ }^{58}$ R. Singh and G. Bester, Phys. Rev. Lett. 103, 063601 (2009).

${ }^{59}$ V. Mlinar and A. Zunger, Phys. Rev. B 79, 115416 (2009).

${ }^{60}$ T. Takagahara, Phys. Rev. B 62, 16840 (2000).

${ }^{61}$ C. Pryor, J. Kim, L. W. Wang, A. J. Williamson, and A. Zunger, J. Appl. Phys. 83, 2548 (1998). 